\title{
An enhanced second carrier harmonic cancellation method for multi-source DC electric power systems
}

\author{
Cheng Wang, Tao Yang, Serhiy Bozhko \\ Power Electronics, Machines and Control Research Group \\ The University of Nottingham \\ Nottingham, UK \\ cheng.wang@nottingham.ac.uk
}

\author{
Ponggorn Kulsangcharoen \\ Motor Drive Systems Centre (MDSC) \\ Collins Aerospace \\ Shirley, Solihull, UK \\ ponggorn.kulsangcharoen@utas.utc.com
}

\begin{abstract}
Multi-source DC power systems have been widely used in electric transportations, including more-electric aircraft, electric ship and electric vehicles. These systems normally involve in power electronic converters whose switching actions may cause current fluctuation on DC-bus capacitors. Eliminating certain order harmonics can help the system potentially reduce the volume and weight of the capacitor. In this paper, a simplified model to estimate 2 nd carrier harmonic of de current in two-level converters is proposed. In derived model, magnitude of the harmonic is only determined by the DC-bus current and modulation index of the converter. Meanwhile, the phase angle of it is determined by angle of carrier signals. Based on this model, a new harmonic cancellation method is proposed. The method has high robustness and can work under any fundamental frequency and power sharing ratio. Simulation results are presented in this paper to verify the validation of proposed harmonic model and the enhanced cancellation method.
\end{abstract}

\section{INTRODUCTION}

With the development of transportation electrification, multi-source DC power systems start to penetrate in hybrid vehicle[1], shipboard[2], and more electric aircraft[3],[4]. The multi-source systems give system advantages such as high redundancy, high flexibility of power management and so on. Fig 1 shows a simple multi-source power system. In the system, there are starter-generators (SG) supplying one common DC bus and give power to load together. Using different control algorithm in controller, optimization targets can be achieved in terms of efficiency, power quality, and reliability.

In the system, switching actions of converters cause current pulses injected into DC-bus capacitor. These pulses will significantly determine the weight, volume, and cost of capacitor. To overcome this problem, research on reducing current pulses on capacitor should be investigated.

In recent years, researchers have published several papers about capacitor reduction for single converter. In [5], mathematical model of DC-link current was developed using double Fourier solution. It gave researchers a potential to analyse harmonics and minimize it. In [6], lower capacitor harmonics are achieved by applying high modulation on DC$\mathrm{AC}$ converter using an additional DC-DC converter. More switching devices are used in the system and the control becomes more complicated. In [7], capacitor ripple current was reduced by applying nonadjacent switching vectors.

For multi-source DC power system, there are few paper published about reducing capacitor. Fortunately, back-to-back converter is similar to multi-source power system. One rectifier and one inverter share same DC-bus capacitor. Meanwhile, fundamental frequencies of inverter and rectifier are not same. Methods of eliminating the switching frequency components of the first order and the second order on DC-bus capacitor were investigated in [8] and [9]. However, the performance of reducing harmonic highly relies on performance of band-pass filter, which limits the application of this method.

In this paper, the research is about cancellation method for the second carrier harmonics on capacitor. Firstly, a simplified mathematical model of second carrier harmonics on DC-bus is investigated. Based on it, a new method on minimizing 2nd carrier harmonic is investigated. This approach can significantly minimize harmonics under different fundamental frequencies and power sharing ratio. Compared with method proposed in [9], measurement on phase angle of harmonic on DC bus is not needed, which makes this method easily applied on low communication bandwidth system. At last, the proposed method has been validated by simulation, and the results are given in Section IV.

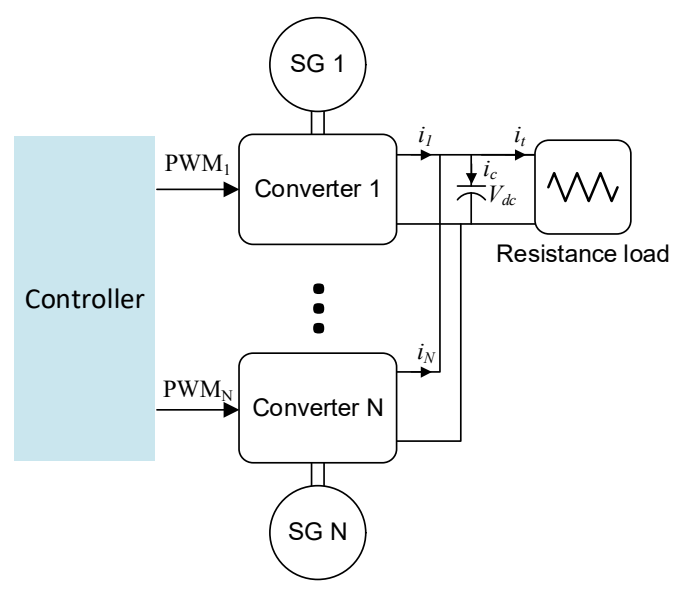

Fig. 1. Multi-source power system 


\section{2ND CARRIER HARMONICS ANALYSIS OF SINGLE CONVERTER}

To eliminate 2nd carrier harmonics, analytical solution for harmonics generated from single converter should be investigated firstly. The 3-phase 2-level converters have been widely used in AC-DC conversion, as shown in Fig. 2. Analysis of the DC-bus current $\left(i_{d c}\right)$ before capacitor will be introduced in this section.

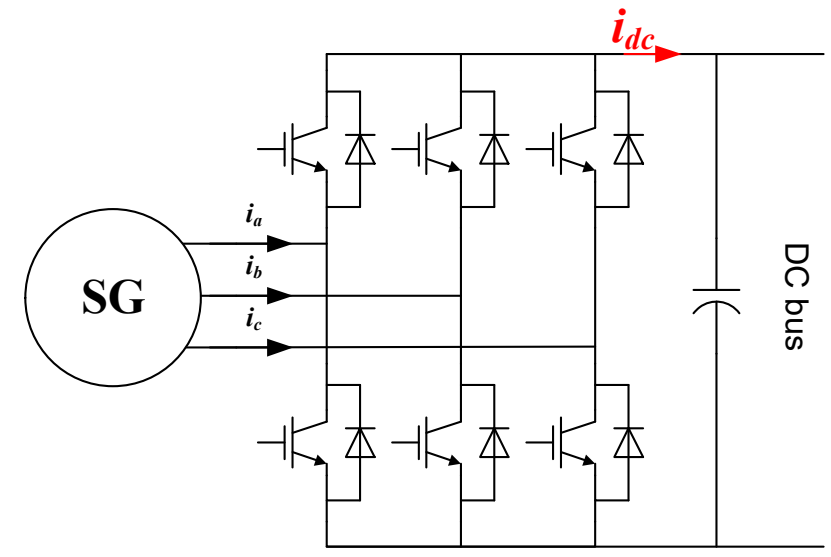

Fig. 2. 2-level converter.

\section{A. Mathematical analysis on DC-bus 2 nd carrier harmonic}

Ignoring switching-frequency fluctuation on $\mathrm{AC}$ side current, for a two-level converter, AC side current is as follows

$$
i_{a c}^{[k]}(t)=I_{a c} \cos \left(2 \pi f_{0} t+\beta+\frac{2 k \pi}{3}\right)
$$

Where $I_{a c}$ is the amplitude of fundamental component of AC current, $f_{0}$ is the fundamental frequency of $i_{a b c}, \beta$ is the angle between phase current and its back-EMF, $k=0,1$ and 2 is to represent phase $\mathrm{A}, \mathrm{B}$ and $\mathrm{C}$.

The current on DC bus is flow from AC side through power converter. Using illustration in [9], for asymmetrical regular sampling PWM, the switching function for one phase leg can be expressed by

$$
\begin{aligned}
s f^{[k]}(t)=K_{0,1} \cos & \left(2 \pi f_{0} t+\beta+\alpha+\frac{2 k \pi}{3}\right) \\
& +\sum_{m=1}^{\infty} \sum_{n=-\infty}^{\infty} K_{m, n} \cos \left[m \left(2 \pi f_{c} t\right.\right. \\
& \left.+\theta_{c}^{[k]}\right)+n\left(2 \pi f_{0} t+\beta+\alpha\right. \\
& \left.\left.+\frac{2 k \pi}{3}\right)\right]
\end{aligned}
$$

Where $f_{c}$ is the switching frequency, $\theta_{c}^{[k]}$ is PWM carrier angle for each leg, $\alpha$ is the phase angle between $\mathrm{AC}$ fundamental current and AC-side converter voltage (power factor angle), $K_{m, n}$ is the harmonic amplitude using Bessel function of first kind. Based on double Fourier analysis[10], $K_{m, n}$ can be expressed by

$$
\begin{gathered}
K_{m, n}=\frac{1}{q} J_{n}\left(q_{m, n} M\right) \sin \left[(m+n) \frac{\pi}{2}\right] \\
q_{m, n}=\left(m+n \frac{f_{0}}{f_{c}}\right) \frac{\pi}{2}
\end{gathered}
$$

Here, function $J_{n}()$ is Bessel function of the first kind. In (3) and (4), $m$ and $n$ are orders of main and band side harmonic. For instance, when $m=1$ and $n=3, K_{m, n}$ means the magnitude of harmonic component on frequency $f_{c}+3 f_{0}$. Using (1) - (4), the DC-bus harmonic currents generated from one phase leg can be derived as

$$
\begin{aligned}
i_{d c}^{[k]}(t)=i_{a c}^{[k]}(t) s & f^{[k]}(t) \\
& =\frac{I_{a c}}{2}\left\{K _ { 0 , 1 } \left[\operatorname { c o s } \left(4 \pi f_{0} t+2 \beta\right.\right.\right. \\
& \left.\left.+\alpha+\frac{4 k \pi}{3}\right)+\cos \alpha\right] \\
& +\sum_{m=1}^{\infty} \sum_{n=-\infty}^{\infty} K_{m, n}\left\{\operatorname { c o s } \left[2 \pi \left(m f_{c}\right.\right.\right. \\
& \left.\left.+(n+1) f_{0}\right) t+\sigma_{m, n}^{[k]}\right] \\
& +\cos \left[2 \pi\left(m f_{c}+(n-1) f_{0}\right) t\right. \\
& \left.\left.\left.+\varphi_{m, n}^{[k]}\right]\right\}\right\}
\end{aligned}
$$

Where $\sigma_{m, n}^{[k]}$ and $\varphi_{m, n}^{[k]}$ are phase angles of each component, which are

$$
\begin{aligned}
& \sigma_{m, n}^{[k]}=m \theta_{c}^{[k]}+(n+1)\left(\beta+\frac{2 k \pi}{3}\right)+n \alpha \\
& \varphi_{m, n}^{[k]}=m \theta_{c}^{[k]}+(n-1)\left(\beta+\frac{2 k \pi}{3}\right)+n \alpha
\end{aligned}
$$

Current harmonics on DC-bus should be expressed as sum of 3 legs' harmonics, which is

$$
\begin{aligned}
i_{d c, i, j}(t)=\frac{I_{a c}}{2} K_{i, j-1} & \sum_{k=0}^{2} \cos \left[2 \pi\left(i f_{c}+j f_{0}\right) t\right. \\
& \left.+\sigma_{i, j-1}^{[k]}\right] \\
& +\frac{I_{a c}}{2} K_{i, j+1} \sum_{k=0}^{2} \cos \left[2 \pi \left(i f_{c}\right.\right. \\
& \left.\left.+j f_{0}\right) t+\varphi_{i, j+1}^{[k]}\right]
\end{aligned}
$$

Where $i$ and $j$ mean orders of main and band side harmonic of current harmonics. This is different from $m$ and $n$ which are orders for switching function.

Phase angle of components in (8) are

$$
\begin{aligned}
& \sigma_{i, j-1}^{[k]}=i \theta_{c}^{[k]}+j\left(\beta+\frac{2 k \pi}{3}\right)+(j-1) \alpha \\
& \varphi_{i, j+1}^{[k]}=i \theta_{c}^{[k]}+j\left(\beta+\frac{2 k \pi}{3}\right)+(j+1) \alpha
\end{aligned}
$$


Consider no phase shift on carrier signals among 3 legs $\left(\theta_{c}^{[1]}=\theta_{c}^{[2]}=\theta_{c}^{[3]}\right)$, as currents from 3 phase legs converge on DC bus together, harmonic generated from 3 legs are cancelled on DC-bus except $\mathrm{j}=0, \pm 3, \pm 6$, etc. This is because of $2 \pi / 3$ phase difference among them.

Apart from phase angle of each component. Magnitudes of components can be derived as

$$
\begin{aligned}
& \frac{I_{a c} K_{i, j-1}}{2}=\frac{I_{a c}}{2 q_{i, j-1}} J_{j-1}\left(q_{i, j-1} M\right) \sin \left[(i+j-1) \frac{\pi}{2}\right] \\
& \frac{I_{a c} K_{i, j+1}}{2}=\frac{I_{a c}}{2 q_{i, j+1}} J_{j+1}\left(q_{i, j+1} M\right) \sin \left[(i+j+1) \frac{\pi}{2}\right]
\end{aligned}
$$

For magnitude unequal to zero, $i+j$ should be even number, otherwise the magnitude will be zero because $\sin [(i+j-1) \pi / 2]$ or $\sin [(i+j+1) \pi / 2]$ equal zero.

$$
\begin{gathered}
j=0, \pm 3, \pm 6 \ldots \\
i+j=\text { even number }
\end{gathered}
$$

Summarized from above illustration, non-zero harmonics can only be achieved when

Hence, $f_{c} \pm 3 f_{0}$ and $2 f_{c}$ appear on DC bus significantly. In this paper, cancellation of $2 f_{c}$ component is focused, and this component can be derived as

$$
\begin{aligned}
i_{d c, 2,0}^{[k]}(t)=\frac{I_{a c}}{2}\{ & K_{2,-1} \cos \left[4 \pi f_{c} t+2 \theta_{c}-\alpha\right] \\
& \left.+K_{2,1} \cos \left[4 \pi f_{c} t+2 \theta_{c}+\alpha\right]\right\}
\end{aligned}
$$

\section{B. Simplified model on 2nd carrier harmonic}

Seen from (15), there are two components with same frequency but different magnitude and phase angle. Using magnitude-angle form, these two components can be expressed as

$$
\frac{I_{a c} K_{2,-1}}{2} \angle\left(2 \theta_{c}-\alpha\right) \text { and } \frac{I_{a c} K_{2,1}}{2} \angle\left(2 \theta_{c}+\alpha\right)
$$

Look into parameter $K_{2,-1}$ and $K_{2,1}$ firstly,

$$
\begin{gathered}
K_{2,1}=-\frac{2}{\left(2+\frac{f_{0}}{f_{c}}\right) \pi} J_{1}\left(\frac{\pi}{2}\left(2+\frac{f_{0}}{f_{c}}\right) M\right) \\
K_{2,-1}=\frac{2}{\left(2-\frac{f_{0}}{f_{c}}\right) \pi} J_{-1}\left(\frac{\pi}{2}\left(2-\frac{f_{0}}{f_{c}}\right) M\right) \\
=-\frac{2}{\left(2-\frac{f_{0}}{f_{c}}\right) \pi} J_{1}\left(\frac{\pi}{2}\left(2-\frac{f_{0}}{f_{c}}\right) M\right)
\end{gathered}
$$

In general, $f_{c}$ is much larger than $f_{0}$. Hence term $f_{0} / f_{c}$ is approximately equal to 0 . Substituting $f_{0} / f_{c}=0$ in to (17) and (18) makes

$$
\begin{gathered}
K_{2,1} \approx K_{2,-1} \approx-\frac{1}{\pi} J_{1}(\pi M) \\
I_{d c, 2,0}(t) \approx-\frac{3 I_{a c} \cos \alpha}{\pi} J_{1}(\pi M) \cos \left(4 \pi f_{c} t+2 \theta_{c}\right)
\end{gathered}
$$

For given output converter power $P$, the AC-side terminal real power of the two-level converter can be formulated as

$$
P=3 \frac{I_{a c}}{\sqrt{2}} \frac{V_{a c}}{\sqrt{2}} \cos \alpha=\frac{3 I_{a c} M V_{d c}}{4} \cos \alpha
$$

Where $V_{a c}$ is magnitude of converter phase voltage, $M$ is modulation index under flux-weakening control. Extract term $I_{a c} \cos \alpha$ from (21) and substitute it into (20), 2nd carrier component can be further expressed as

$$
\begin{gathered}
I_{d c, 2,0}(t) \approx \frac{4 P J_{1}(\pi M)}{\pi M V_{d c}} \cos \left(4 \pi f_{c} t+2 \theta_{c}\right) \\
=\frac{4 I_{d c} J_{1}(\pi M)}{\pi M} \cos \left(4 \pi f_{c} t+2 \theta_{c}\right)
\end{gathered}
$$

Where $I_{d c}$ is the value of DC component current on DCbus. In (22), the simplified model also eliminates the influence from power factor angle $\alpha$, which is always determined by many elements such as rotor speed, output power, and so on. Magnitude of 2nd carrier harmonic current is only influenced by $I_{d c}$ and $M$. Meanwhile, phase angle of the component is only determined by $\theta_{c}$.

Fig 3 shows the vector diagram of simplification work. Term 1 and 2 in black represent components in (16). Using simplification process from (19) to (22), term 1 and 2 reform to same magnitude which is shown in red. The simplified synthesized vector also has a fixed phase angle which is $2 \theta_{c}$. In Fig 3, the difference between synthesized vectors in blue and red is obviously visible. However, this is drawn for clear illustration. In practical, the difference is quite small due to $f_{0} / f_{c}$ is approximately equal to 0 . This will also be proved in next subsection.

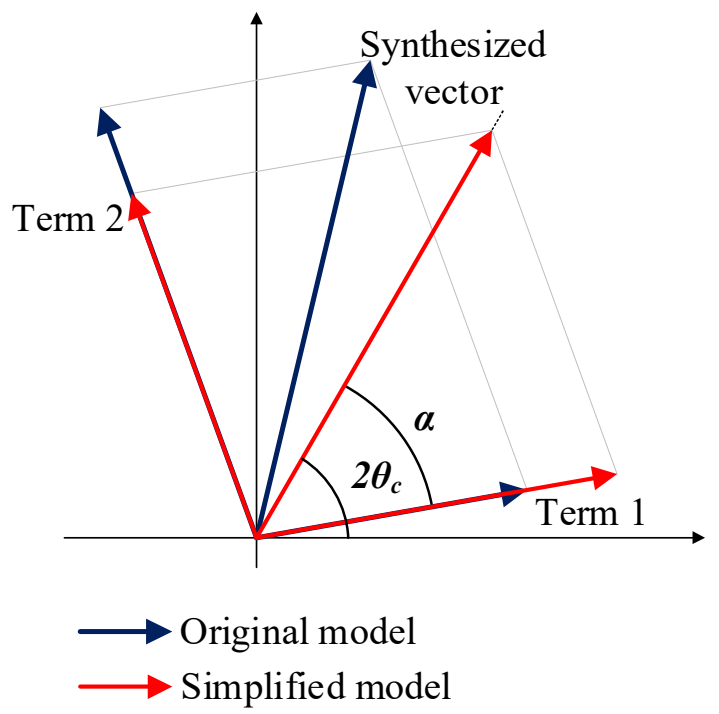

Fig. 3. Vector diagram of simplification.

\section{Comparison between simplified and original model}

The comparison between original (15) and simplified (22) model is given in Fig 4. Fig $4 \mathrm{a}$ and $\mathrm{b}$ show the accurate 
magnitude and angle under different rotor speed and output power together with simplified model respectively. The calculation is based on a PMSG power generation system. Modulation index is set as 0.95 . Parameter of PMSG is given in Table I. These two figures shows a higher error when PMSG works under higher speed. This is caused by assuming $f_{0} / f_{c}=0$ in (19). Higher rotor speed means larger $f_{0}$ which makes $f_{0} / f_{c}$ not near 0 as it when under low speed.

Meanwhile, both magnitude and angle show larger error under low power output. Actually, this is a little complicated to analyse the reason because it is caused by power factor angle and magnitudes of two terms in (16) together. There are too many interaction relations from machine parameter and control scheme.

However, it is obvious that magnitude under low power is not significant. That means it will affect less on DC-bus current harmonics compared with that under higher power. Hence, this error can be ignored in practical, because the control system only care about the worst case which is high power operation.

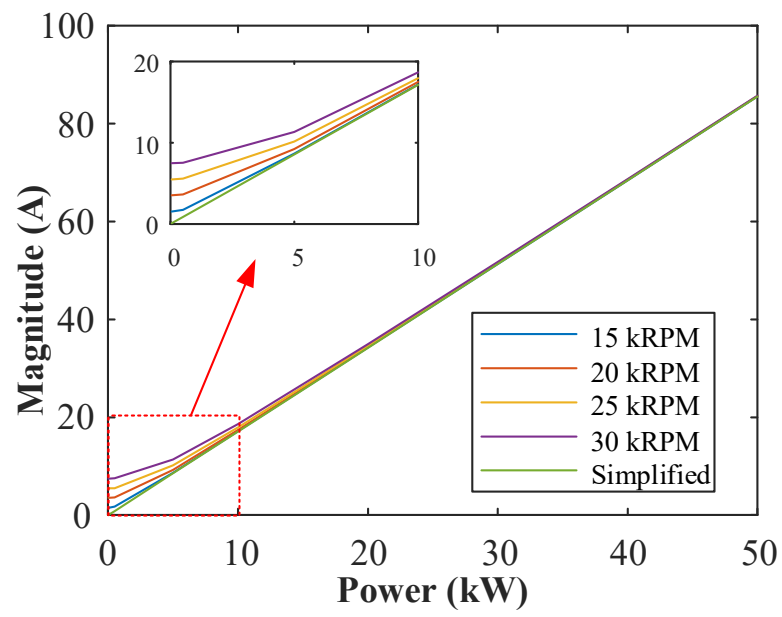

(a)

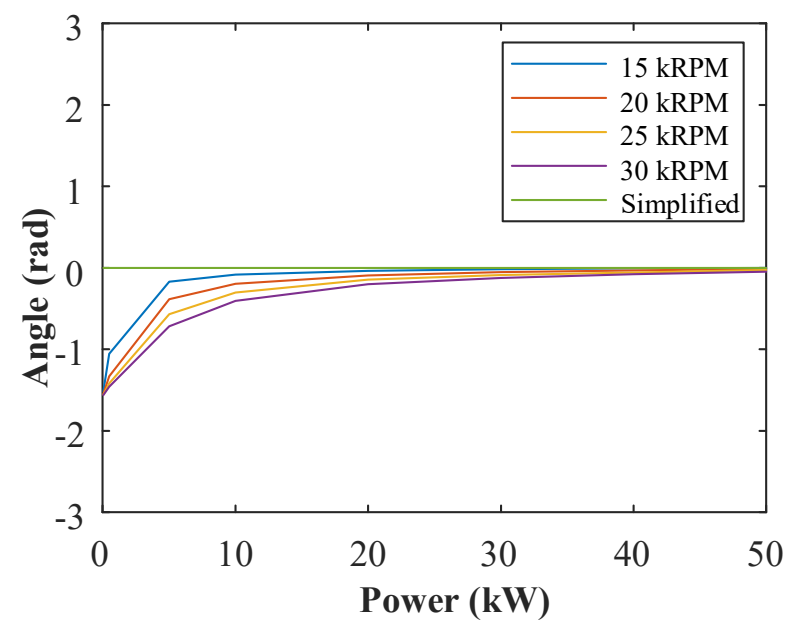

(b)

Fig. 4. Vector diagram of simplification. a) Magnitude, b) Angle.
TABLE I. PMSG PARAMETERS

\begin{tabular}{|c|c|}
\hline Parameters & Value \\
\hline Number of poles & 6 \\
\hline Machine resistance & $1.058 \mathrm{~m} \Omega$ \\
\hline Machine d-axis inductance & $99 \mu \mathrm{H}$ \\
\hline Machine q-axis inductance & $99 \mu \mathrm{H}$ \\
\hline Flux linkage of permanent magnet & $0.03644 \mathrm{Vs} / \mathrm{rad}$ \\
\hline
\end{tabular}

\section{PROPOSED METHOD}

Using simplified model illustrated in previews section, a new 2nd carrier harmonic cancellation method for multisource system was proposed in this section.

\section{A. Method for 1:1 power sharing ratio}

A system only contains 2 sources and one resistance load was researched in this section, which is shown in Fig 5. The purpose of controller is to adjust DC bus voltage constant at reference value $V_{\text {ref. }}$ A centralized controller is applied to realize power sharing control and PWM generation. In practical, controller can also be decentralized controller with information communication. Using centralized controller here is just for simple explanation.

The main concept of proposed method is 2nd carrier harmonics generated from converters interactively cancel each other. It means two components has same magnitude but 180-degree phase shift. When two sources work in average, $I_{d c l}=I_{d c 2}$. Seen from (22), same magnitudes can be achieved when applying same modulation index on both sources. Meanwhile, phase angle difference $\left(2 \theta_{c}^{\{1\}}-2 \theta_{c}^{\{2\}}\right)$ should be 180 degrees, which means phase difference between two carrier signals $\left(\theta_{c}^{\{1\}}-\theta_{c}^{\{2\}}\right)$ should be 90 degrees. Here, $\{1\}$ and $\{2\}$ mean the index of SG.

The control diagram was shown in Fig 5. A centralized voltage controller works for making DC bus voltage following reference. DC current reference is generated and broadcast to each PWM generator, hence power sharing between two converters is in average. Then, by a 90-degree phase shift on carrier signal, harmonic cancellation can be achieved.

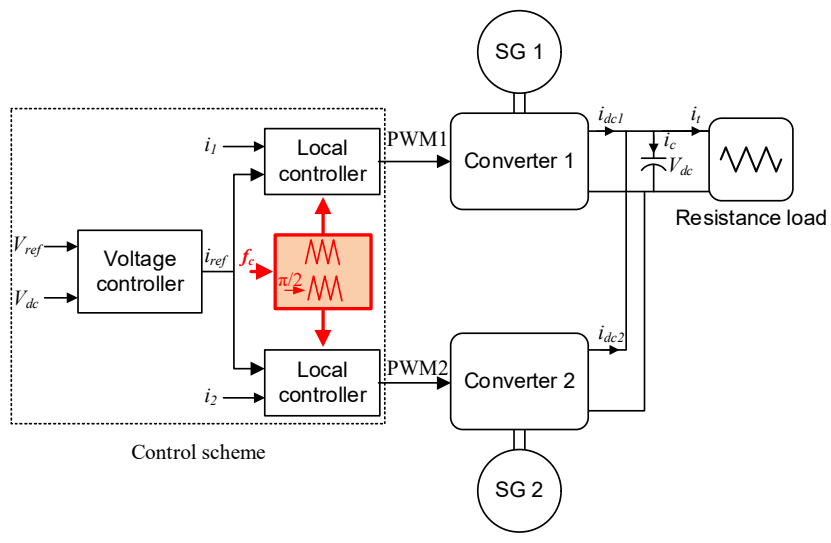

Fig. 5. Control diagram of the proposed method. 


\section{B. Method for other power sharing ratio}

In practical system, non-average power sharing ratio was always applied according to different optimizing objects. Only applying 90-degree phase shift cannot eliminate each other totally because the magnitudes of 2 components are different. To overcome this problem, a new control scheme was proposed and shown in Fig 6. Between the local controllers and voltage controller, an additional 2nd carrier harmonic controller block was applied. This block is to calculate the individual current references and modulation index based on power sharing ratio and maximum modulation index. Firstly, define $K$ as power sharing ratio between two sources which is

$$
K=\frac{i_{\text {ref } 1}}{i_{\text {ref } 2}}
$$

Hence, the exact value of $i_{r e f 1}$ and $i_{\text {ref } 2}$ can be expressed as

$$
\left\{\begin{array}{l}
i_{r e f 1}=i_{r e f} \frac{K}{K+1} \\
i_{r e f 2}=i_{r e f} \frac{1}{K+1}
\end{array}\right.
$$

Then, same magnitudes of 2 nd carrier harmonics should be achieved. Seen from (22), the relation should be

$$
\frac{4 i_{r e f 1} J_{1}\left(\pi M_{1}\right)}{\pi M_{1}}=\frac{4 i_{r e f 2} J_{1}\left(\pi M_{2}\right)}{\pi M_{2}}
$$

Modulation index of each converter can be manipulated to achieve same 2 nd carrier harmonic magnitude between two sources. Smaller modulation index can increase the magnitude which is shown in (22). Assuming $K<1$, which means SG2 produce more power than SG1, and it should operate under maximum modulation index $M_{\max } . M_{l}$ is manipulated for 2 nd carrier harmonic to reach the same magnitude value, which is

$$
\frac{J_{1}\left(\pi M_{1}\right)}{M_{1}}=\frac{J_{1}\left(\pi M_{\max }\right)}{M_{\max }}
$$

Define function

$$
f(M)=\frac{J_{1}(\pi M)}{M}
$$

Manipulated $M_{l}$ can be derived using inverse function of (27) which is

$$
M_{1}=f^{-1}\left(\frac{J_{1}\left(\pi M_{\max }\right)}{K M_{\max }}\right)
$$

However this inverse function is hard to calculate. Hence, a look-up table is applied in practical. Fig 7 shows the relation between $M$ and $K$ when $M_{\max }=0.9$ and 0.95 respectively. A lower limitation 0.5 was set to prevent high negative d-axis current, which will generate higher loss.

When $\mathrm{K}>1$, same solution can be implemented. Modulation index for both sources are

$$
M_{1}=1 \text { and } M_{2}=f^{-1}\left(\frac{K J_{1}\left(\pi M_{\max }\right)}{M_{\max }}\right)
$$

Using manipulated modulation index together with 90 degree phase shift on carrier signal, 2nd carrier harmonics on DC-bus capacitor can be supressed under non-average power sharing ratio.

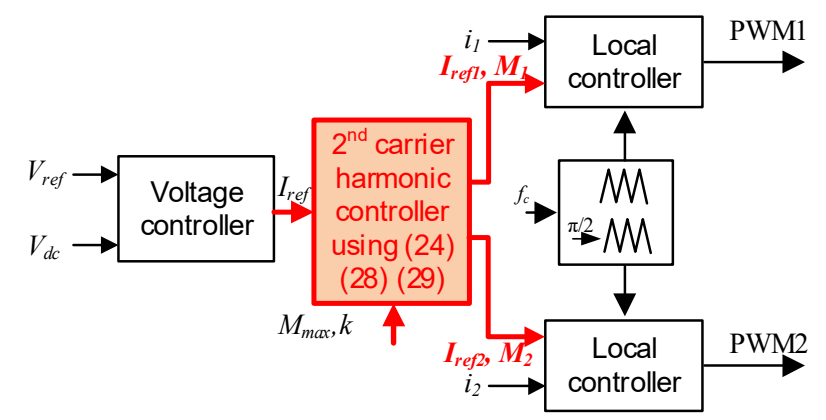

Fig. 6. Control diagram of 2nd carrier harmonic cancellation.

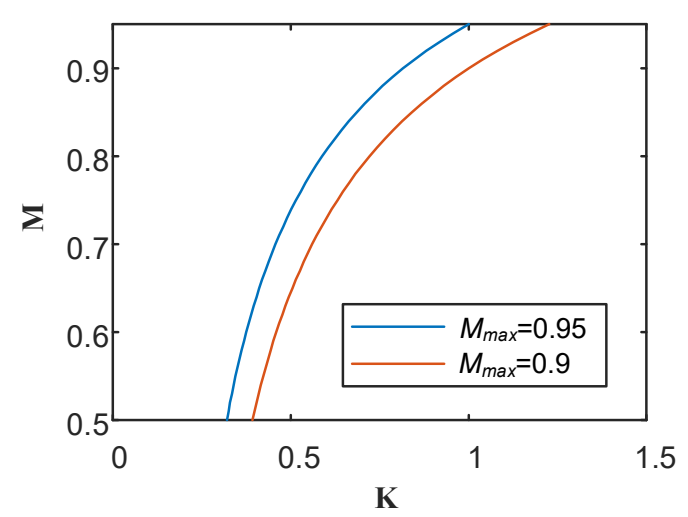

Fig. 7. M-K look-up figure.

\section{Method for system with more than two sources}

This paper focuses on dual-channel system. The simplified can also be utilized for system with more than two converters with same basic concept. However, this is not the important part of this paper, and will be presented in future work.

\section{SIMULATON RESULTS}

Simulations based on MATLAB/Simulink and PLECS was implemented to evaluate the performance of proposed harmonic model and cancellation method. Some basic control parameters for multi-source power system was shown in Table II.

TABLE II. SYSTEM CONTROL PARAMETER

\begin{tabular}{|c|c|}
\hline Parameters & Value \\
\hline DC-bus capacitance & $200 \mu \mathrm{F}$ \\
\hline Voltage reference & $270 \mathrm{~V}$ \\
\hline Load resistance & $1.35 \Omega$ \\
\hline Rotor speed & $20 \mathrm{kRPM}, 30 \mathrm{kRPM}$ \\
\hline Switching frequency & $32 \mathrm{kHz}$ \\
\hline Maximum modulation index & 0.95 \\
\hline
\end{tabular}




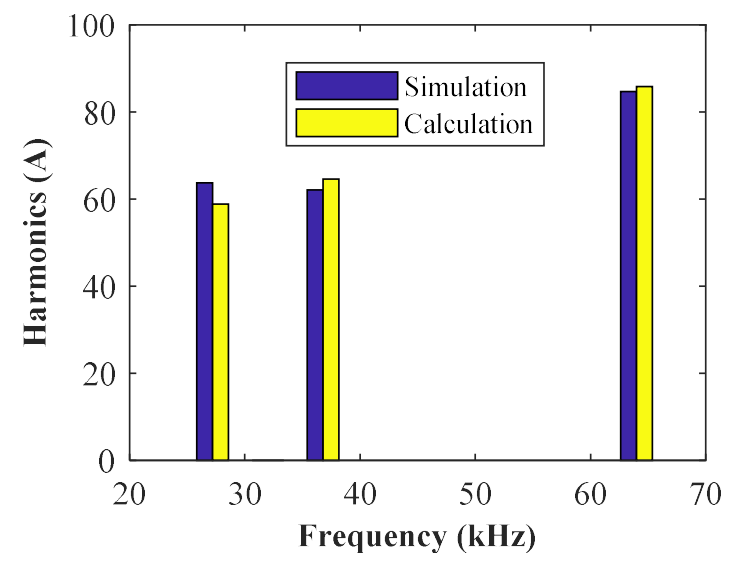

Fig. 8. Comparison between mathematical model and simulation.
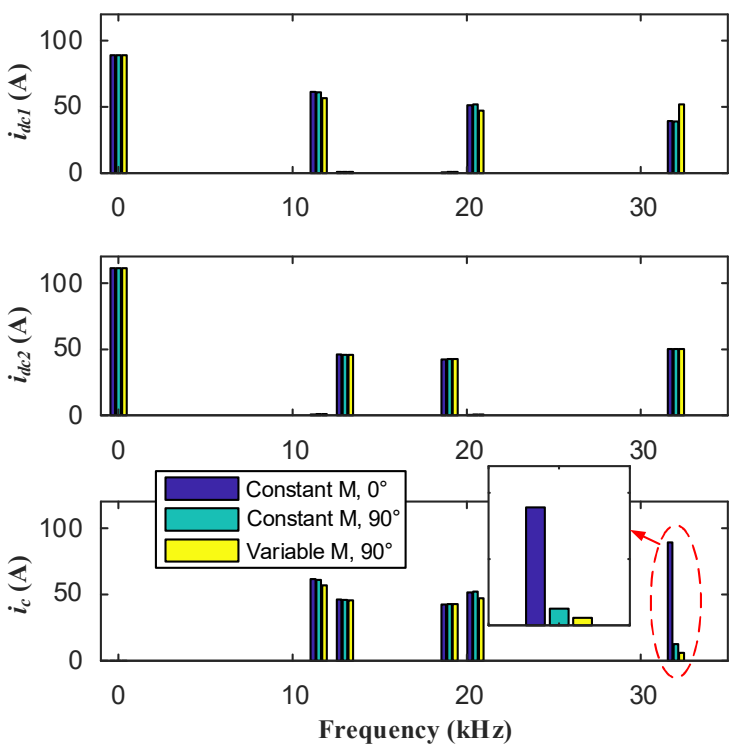

Fig. 9. Current spectrums when power sharing ratio is 4:5.

For harmonics generated from single converter, Fig 8 shows the spectrum comparison between simulation and calculation results. It shows a well match between each other. Only harmonics on $f_{c} \pm 3 f_{0}$ and $2 f_{c}$ are significant.

Fig 9 shows the capacitor current spectrums when power sharing ratio is 4:5. Currents under three operations are shown together, which is a) constant $\mathrm{M}(0.95)$ with no phase shift, b) constant $M$ with 90 degrees phase shift, and c) variable $M$ with 90 degrees phase shift. It can be seen that constant modulation index with 90 degrees phase shift has significantly suppressed the value of 2 nd carrier harmonic on DC-bus capacitor. What's more, with variable modulation index, the component is further suppressed, which validate the harmonic cancellation under non-average power sharing ratio.

\section{CONCLUSION}

In this paper, a simplified mathematical model on $2^{\text {nd }}$ carrier current harmonic was investigated firstly. The results show the magnitude of component was only determined by the value of DC current and modulation index, while phase angle of it is caused by carrier phase angle.

Based on simplified model, a new 2nd harmonic cancellation method was proposed. By manipulating modulation index together with 90 -degree phase shift on carrier signal, cancellation can be realized in a 2-source power system. The method can work under any machine speed and need no communication of phase angle among sources.

Finally, simulation has been done to verify the validation of the proposed 2 nd harmonic model and cancellation method.

\section{REFERENCES}

[1] T. A. Burress, S. L. Campbell, C. Coomer, C. W. Ayers, A. A. Wereszczak, J. P. Cunningham, L. D. Marlino, L. E. Seiber, and H.-T. Lin, Evaluation of the 2010 Toyota Prius Hybrid Synergy Drive System. No. March, 2011

[2] B. Z. Jin, G. Sulligoi, R. Cuzner, L. Meng, J. C. Vasquez, and J. M. Guerrero, "Next-Generation Shipboard DC Power System," pp. 45-57, 2016.

[3] F. Gao, Decentralised Control and Stability Analysis of a MultiGenerator Based Electrical Power System for More Electric Aircraft. No. August, 2016

[4] F. Gao, S. Bozhko, G. Asher, P. Wheeler, and C. Patel, "An Improved Voltage Compensation Approach in a Droop-Controlled DC Power System for the More Electric Aircraft," IEEE Transactions on Power Electronics, vol. 31, no. 10, pp. 7369-7383, 2016.

[5] B. P. McGrath and D. G. Holmes, "A general analytical method for calculating inverter DC-link current harmonics," IEEE Transactions on Industry Applications, vol. 45, no. 5, pp. 1851-1859, 2009.

[6] F. D. Kieferndorf, M. Förster, and T. A. Lipo, "Reduction of DC-bus capacitor ripple current with PAM/PWM converter," IEEE Transactions on Industry Applications, vol. 40, no. 2, pp. 607-614, 2004.

[7] T. D. Nguyen, N. Patin, and G. Friedrich, "Extended double carrier PWM strategy dedicated to RMS current reduction in DC link capacitors of three-phase inverters," IEEE Transactions on Power Electronics, vol. 29, no. 1, pp. 396-406, 2014.

[8] L. Shen, S. Bozhko, G. Asher, C. Patel, and P. Wheeler, "Active DC Link Capacitor Harmonic Current Reduction in Two-Level Back-toBack Converter," IEEE Transactions on Power Electronics, vol. 31, no. 10, pp. 6947-6954, 2016.

[9] L. Shen, S. Bozhko, C. I. Hill, and P. Wheeler, "DC-Link Capacitor Second Carrier Band Switching Harmonic Current Reduction in TwoLevel Back-to-Back Converters," IEEE Transactions on Power Electronics, vol. 33, no. 4, pp. 3567-3574, 2018.

[10] T. Lipo and D. G. Holmes, Pulse Width Modulation for Power Converters. 2003. 